TRANSACTIONS OF THE

AMERICAN MATHEMATICAL SOCIETY

Volume 358, Number 2, Pages 671-685

S 0002-9947(05)03637-8

Article electronically published on March 10, 2005

\title{
SOME QUOTIENT HOPF ALGEBRAS OF THE DUAL STEENROD ALGEBRA
}

\author{
J. H. PALMIERI
}

\begin{abstract}
Fix a prime $p$, and let $A$ be the polynomial part of the dual Steenrod algebra. The Frobenius map on $A$ induces the Steenrod operation $\widetilde{P}^{0}$ on cohomology, and in this paper, we investigate this operation. We point out that if $p=2$, then for any element in the cohomology of $A$, if one applies $\widetilde{P}^{0}$ enough times, the resulting element is nilpotent. We conjecture that the same is true at odd primes, and that "enough times" should be "once."

The bulk of the paper is a study of some quotients of $A$ in which the Frobenius is an isomorphism of order $n$. We show that these quotients are dual to group algebras, the resulting groups are torsion-free, and hence every element in Ext over these quotients is nilpotent. We also try to relate these results to the questions about $\widetilde{\mathscr{P}} 0$. The dual complete Steenrod algebra makes an appearance.
\end{abstract}

\section{INTRODUCTION}

Fix a prime number $p$, and let $A$ be the following familiar Hopf algebra: as an algebra,

$$
A=\mathbf{F}_{p}\left[\xi_{1}, \xi_{2}, \xi_{3}, \ldots\right]
$$

and the coproduct is determined by

$$
\Delta\left(\xi_{n}\right)=\sum_{i=0}^{n} \xi_{n-i}^{p^{i}} \otimes \xi_{i}
$$

where $\xi_{0}=1$. There is also an antipode (see Lemma 3.6) but it does not play a central role here. $A$ is graded by putting $\xi_{n}$ in degree $2^{n}-1$ when $p=2$, and in degree $2 p^{n}-2$ when $p$ is odd. This makes $A$ into a graded connected commutative Hopf algebra.

When $p=2$, the graded dual of $A$ is the mod 2 Steenrod algebra; when $p$ is odd, the graded dual of $A$ is the algebra of reduced power operations, which is a quotient of the $\bmod p$ Steenrod algebra. We will occasionally abuse the language and refer to $A$ as the dual Steenrod algebra, regardless of the prime. We write $A^{*}$ for its graded dual.

Homotopy theorists would like to understand the cohomology of $A$, which can be defined as $\operatorname{Ext}_{A^{*}}^{* *}\left(\mathbf{F}_{p}, \mathbf{F}_{p}\right)$, where Ext is computed in the category of graded $A^{*}$ modules, or equivalently as $\operatorname{Ext}_{A}^{* * *}\left(\mathbf{F}_{p}, \mathbf{F}_{p}\right)$, where Ext is computed in the category

Received by the editors January 7, 2003 and, in revised form, February 19, 2004.

2000 Mathematics Subject Classification. Primary 55S10, 18G15, 20E18, 20J06.

(C)2005 American Mathematical Society 
of graded $A$-comodules. (In places where it makes a difference, we will use the comodule definition.) Note that in this setting, Ext is bigraded: the first grading is the usual homological one, and the second is induced by the grading on the (co)module category. The Frobenius map $a \mapsto a^{p}$ on $A$ is a Hopf algebra map, and so it induces an algebra map on cohomology. We denote this map by $\widetilde{\mathscr{P}}^{0}$ when $p$ is unspecified or odd, and by $\widetilde{S q}^{0}$ when $p=2$ (see Proposition 5.1 for an explanation of this notation). Since the Frobenius does not preserve the grading, neither does $\widetilde{\mathscr{P}^{0}}$ _it works like this:

$$
\widetilde{\mathscr{P}^{0}}: \operatorname{Ext}_{A}^{s, t}\left(\mathbf{F}_{p}, \mathbf{F}_{p}\right) \rightarrow \operatorname{Ext}_{A}^{s, p t}\left(\mathbf{F}_{p}, \mathbf{F}_{p}\right) .
$$

We would like to understand how the map $\widetilde{\mathscr{P}}^{0}$ interacts with the multiplicative structure of $\operatorname{Ext}_{A}^{*}\left(\mathbf{F}_{p}, \mathbf{F}_{p}\right)$. This paper has two goals: to advertise some questions and conjectures related to this interaction, and to study some Hopf algebras related to $A$ that arise when studying $\widetilde{\mathscr{P}}^{0}$ and the Frobenius.

We start by pointing out that

$$
\widetilde{\mathscr{P}^{0}}: \operatorname{Ext}_{A}^{0,0}\left(\mathbf{F}_{p}, \mathbf{F}_{p}\right) \rightarrow \operatorname{Ext}_{A}^{0,0}\left(\mathbf{F}_{p}, \mathbf{F}_{p}\right)
$$

is the identity map. Regarding Ext in positive dimensions, one can deduce the following from the main result in [Pal99].

Theorem 1.2. Let $p=2$ and fix $z \in \operatorname{Ext}_{A}^{s, t}\left(\mathbf{F}_{2}, \mathbf{F}_{2}\right)$. If $s$ is positive, then there is an $n$ so that $\left(\widetilde{S q}^{0}\right)^{n}(z)$ is nilpotent.

This fails if $A$ is replaced by the sub-Hopf algebra $\mathbf{F}_{2}\left[\xi_{1}\right]$ - the cohomology of this is $\mathbf{F}_{2}\left[h_{10}, h_{11}, h_{12}, \ldots\right]$, with $\widetilde{S q}^{0}\left(h_{1, n}\right)=h_{1, n+1}$-but holds if $A$ is replaced by any quotient Hopf algebra. See the appendix for the theorem's proof. One might ask whether there is a simpler proof of this, one which would hold at any prime.

Question 1.3. (a) Is the analogous result true when $p$ is odd?

(b) Can one prove Theorem [1.2 without appealing to the $F$-isomorphism of [Pal99, Theorem 1.2]?

It is also natural to ask, given $z$ as in Theorem 1.2, how large must $n$ be to make $\left(\widetilde{S q}^{0}\right)^{n}(z)$ nilpotent? $\widetilde{S q}$ is an algebra map, so if $z$ is nilpotent, then so is $\widetilde{S q}^{0}(z)$. For every known non-nilpotent element $z$ (except for $z=1$ ), $\widetilde{S q}^{0}(z)$ is nilpotent as well. This leads us to make the following conjecture. All of our evidence is valid only for the prime 2, but we brashly state it for all primes.

Conjecture 1.4. Fix a prime $p$ and an element $z \in \operatorname{Ext}_{A}^{s, t}\left(\mathbf{F}_{p}, \mathbf{F}_{p}\right)$ with $s>0$. Then $\widetilde{\mathscr{P}}^{0}(z)$ is nilpotent.

This is rather specific to $A$ : Theorem 1.2 fails for $\mathbf{F}_{p}\left[\xi_{1}\right]$, and the conjecture implies the theorem. Thus the conjecture does not hold for sub-Hopf algebras of $A$. It also fails for quotient Hopf algebras of $A$ : for example, the cohomology of $\mathbf{F}_{2}\left[\xi_{2}\right] /\left(\xi_{2}^{4}\right)$ is isomorphic to $\mathbf{F}_{2}\left[h_{20}, h_{21}\right]$, with $\widetilde{S q}\left(h_{20}\right)=h_{21}$. (Note, though, that a modified conjecture may hold for quotients of $A$ : given a quotient Hopf algebra $B$ of $A$, there exists an $n$ so that for all $z \in \operatorname{Ext}_{A}^{s, t}\left(\mathbf{F}_{p}, \mathbf{F}_{p}\right)$ with $s>0,\left(\widetilde{\mathscr{P}}^{0}\right)^{n}(z)$ is nilpotent.) 
One can interpret many of these questions in terms of the "double" $\Phi A$ of $A$ : let $\Phi A$ be the sub-Hopf algebra of $A$ generated by the $p$ th powers of the generators:

$$
\Phi A=\mathbf{F}_{p}\left[\xi_{1}^{p}, \xi_{2}^{p}, \xi_{3}^{p}, \ldots\right] .
$$

Then $A$ and $\Phi A$ are isomorphic via the Frobenius map $x \mapsto x^{p}$, and so their Ext algebras are isomorphic. We write $\phi$ for the isomorphism:

$$
\phi: \operatorname{Ext}_{A}^{s, t}\left(\mathbf{F}_{p}, \mathbf{F}_{p}\right) \stackrel{\cong}{\longrightarrow} \operatorname{Ext}_{\Phi A}^{s, p t}\left(\mathbf{F}_{p}, \mathbf{F}_{p}\right) .
$$

Since $\Phi A$ is a sub-Hopf algebra of $A$, there is also an induced map which preserves the grading:

$$
\rho: \operatorname{Ext}_{\Phi A}^{s, t}\left(\mathbf{F}_{p}, \mathbf{F}_{p}\right) \rightarrow \operatorname{Ext}_{A}^{s, t}\left(\mathbf{F}_{p}, \mathbf{F}_{p}\right) .
$$

It is not hard to see that for any $z \in \operatorname{Ext}_{A}^{s, t}\left(\mathbf{F}_{p}, \mathbf{F}_{p}\right)$,

$$
\rho(\phi(z))=\widetilde{\mathscr{P}}^{0}(z) .
$$

So, for example, Conjecture 1.4 is equivalent to the statement that every positivedimensional element in the image of $\rho$ is nilpotent.

One can also reformulate these questions, conjectures, and results as follows. Let $\widehat{A}$ be the dual complete Steenrod algebra: $\widehat{A}$ is the $\mathbf{Z}\left[\frac{1}{p}\right]$-graded Hopf algebra obtained from $A$ by adjoining all $p^{k}$ th roots of each indeterminate $\xi_{n}$ :

$$
\widehat{A}=\underline{\lim }\left(\mathbf{F}_{p}\left[\xi_{n}: n \geq 1\right] \rightarrow \mathbf{F}_{p}\left[\xi_{n}^{p^{-1}}: n \geq 1\right] \rightarrow \mathbf{F}_{p}\left[\xi_{n}^{p^{-2}}: n \geq 1\right] \rightarrow \cdots\right) .
$$

(In the language of Arn94], Arn00], and [LH96, $\widehat{A}$ is the "root algebra" associated to $A$.) The coproduct on $A$ induces one on $\widehat{A}$ :

$$
\Delta\left(\xi_{n}^{p^{k}}\right)=\sum_{i=0}^{n} \xi_{n-i}^{p^{i+k}} \otimes \xi_{i}^{p^{k}}
$$

for all $n \geq 1$ and all $k \in \mathbf{Z}$, where $\xi_{0}=1$ as usual. The antipode for $A$ also induces one for $\widehat{A}$, which we will not need. The dual $\widehat{A}^{*}$ of $\widehat{A}$ is the complete Steenrod algebra, which was introduced by Arnon Arn94, Arn00, and has also been studied by Llerena and Hu'ng [LH96]. (Note that $\widehat{A}^{*}$ is not a graded algebra, since it is larger than the direct sum of its graded pieces; see [LH96, Remark 1.5]. $\widehat{A}$ is a graded algebra, though; the difference is that $\widehat{A}$ is defined as a direct limit, while $\widehat{A}^{*}$ is an inverse limit.)

In other words, one obtains $\widehat{A}$ from $A$ by inverting the Frobenius map. As a result, one can show (see Proposition 5.3) that the cohomology of $\widehat{A}$ is isomorphic to the cohomology of $A$ with the operation $\widetilde{\mathscr{P}} 0$ inverted.

Thus Theorem 1.2 says that every positive dimensional element in $\operatorname{Ext}_{\widehat{A}}^{*}\left(\mathbf{F}_{2}, \mathbf{F}_{2}\right)$ is nilpotent, and Question [1.3(a) asks whether this is true at odd primes. $\widehat{A}$ seems as though it should be easier to understand than $A$. Hence we have the following natural question.

Question 1.5. What is the cohomology of $\widehat{A}$ ?

In order to approach all of these questions, we try something simpler: rather than constructing $\widehat{A}$ by formally inverting the Frobenius and $\widetilde{P}^{0}$, thus making them isomorphisms with infinite order, we will construct Hopf algebras in which they are invertible with finite order. 
Thus we study the following quotient Hopf algebras of $A$ : fix $n \geq 1$ and define $H^{n}$ by

$$
H^{n}=A /\left(\xi_{i}^{p^{n}}-\xi_{i}: i \geq 1\right) .
$$

This is a version of $A$ in which the Frobenius has order $n$. In this paper, we show that the topological linear dual of $H^{n}$ is a group algebra and we identify the group. We use this observation to show that $\operatorname{Ext}_{H^{n}}^{*, *}\left(\mathbf{F}_{p}, \mathbf{F}_{p}\right)$ consists entirely of nilpotent elements. We also try to relate these Ext calculations to the cohomology of the complete Steenrod algebra.

A few words of warning may be warranted for any topologists who may be reading this. First, $H^{n}$ does not inherit the grading from $A$, since the ideal is not homogeneous. Many topologists are used to dualizing quotient Hopf algebras of $A$ to get sub-Hopf algebras of the Steenrod algebra, but since $H^{n}$ is not graded, its dual sits inside the ungraded dual of $A$, which is distinctly different from the usual Steenrod algebra. After all, since $A$ is finite dimensional in each grading, it is isomorphic to its double graded dual; since it is infinite dimensional as a whole, it is not isomorphic to its double ungraded dual.

For example, let $p=2$ and $n=1$. If one dualizes with respect to the monomial basis of $A$, then $\xi_{1}^{i}$ is dual to an element called $\mathrm{Sq}^{i}$. Hence when one dualizes $H^{1}$, since $\xi_{1}$ is identified with $\xi_{1}^{i}$ for all $i$, the element dual to $\xi_{1}$ in $\left(H^{1}\right)^{*}$ deserves to be called $\sum_{j \geq 1} \mathrm{Sq}^{j}$. This element is nonnilpotent - compare this to the graded dual of $A$, in which such infinite sums do not occur and in which every element in the augmentation ideal is nilpotent. The nonnilpotence of $x=\sum_{j \geq 1} \mathrm{Sq}^{j}$ is reflected by the coproduct in $H^{1}: \Delta\left(\xi_{2}\right)$ contains a term $\xi_{1}^{2} \otimes \xi_{1}=\xi_{1} \otimes \xi_{1}$, and hence $x^{2} \neq 0$-it is detected by $\xi_{2} . \Delta\left(\xi_{4}\right)$ has a term $\xi_{2} \otimes \xi_{2}$, so the iterated coproduct of $\xi_{4}$ has a term $\xi_{1} \otimes \xi_{1} \otimes \xi_{1} \otimes \xi_{1}$, and hence $x^{4} \neq 0$. For each $n, \Delta\left(\xi_{2^{n}}\right)$ has a term $\xi_{2^{n-1}} \otimes \xi_{2^{n-1}}$, and hence inductively $x^{2^{n}} \neq 0$ for all $n$.

In other words, the Hopf algebras $H^{n}$ and their duals may challenge the intuition of any readers (or authors) who are used to working with the Steenrod algebra.

Here is an outline of the paper: in Section 2, we show that $H^{n}$ is dual to a group algebra, and we identify the group - it is the group of strict automorphisms of the additive formal group law over $\mathbf{F}_{p^{n}}$. We accomplish this by imitating work of Ravenel and others on the Morava stabilizer groups. We also note that this automorphism group is a torsion-free pro- $p$ group. In Section 3, we collect some observations about $H^{n}$ and the associated automorphism group; for example, we point out that the group is not $p$-adic analytic. In Section 4 we use the fact that the group is torsion-free to show that its cohomology consists entirely of nilpotent elements. In Section 5 we fill in some of the details of the ideas in this introduction: the relationship between the Frobenius on $A$ and the Steenrod operation $\widetilde{\mathscr{P}}^{0}$, and also the completed Steenrod algebra and its cohomology. There is also an appendix which contains the proof of Theorem 1.2 .

Convention. Throughout, an unadorned tensor product $\otimes$ means tensor product over $\mathbf{F}_{p}$.

\section{2. $H^{n}$ IS DUAL TO A GROUP ALGEBRA}

Let $H^{n}$ be as above. Let $B_{j}^{n}$ be the sub-Hopf algebra of $H^{n}$ generated by $\xi_{i}$ with $1 \leq i \leq j$. Let $H_{j}^{n}$ be the quotient of $H^{n}$ by $B_{j}^{n}$. Let $\mathbf{F}=\mathbf{F}_{p^{n}}$. That is, for 
fixed $n \geq 1$ and $j \geq 0$,

$$
\begin{gathered}
H^{n}=\mathbf{F}_{p}\left[\xi_{1}, \xi_{2}, \ldots\right] /\left(\xi_{i}^{p^{n}}-\xi_{i}: i \geq 1\right), \\
H_{j}^{n}=\mathbf{F}_{p}\left[\xi_{j+1}, \xi_{j+2}, \ldots\right] /\left(\xi_{i}^{p^{n}}-\xi_{i}: i \geq j+1\right), \\
B_{j}^{n}=\mathbf{F}_{p}\left[\xi_{1}, \xi_{2}, \ldots, \xi_{j}\right] /\left(\xi_{i}^{p^{n}}-\xi_{i}: j \geq i \geq 1\right), \\
\mathbf{F}=\mathbf{F}_{p^{n}}
\end{gathered}
$$

Note that $H_{0}^{n}=H^{n}$.

In this section, we show that after tensoring with $\mathbf{F}$, each of these Hopf algebras is dual to a group algebra, we identify the groups, and we observe that the group associated to $H_{j}^{n}$ is torsion-free for each $n$ and $j$.

First we should verify that $H_{j}^{n}$ is a quotient Hopf algebra of $A$ for each $n$ and $j$. Given this, it is clear from the formula for the coproduct (1.1) that $B_{j}^{n}$ is a sub-Hopf algebra of $H^{n}$.

Lemma 2.1. Fix $n \geq 1$ and $j \geq 0$. Then

$$
I_{j}^{n}=\left(\xi_{1}, \ldots, \xi_{j} ; \xi_{i}^{p^{n}}-\xi_{i}: i \geq j+1\right)
$$

is a Hopf ideal of $A$. Thus $H_{j}^{n}$ is a quotient Hopf algebra of $A$.

Proof. We have defined $I=I_{j}^{n}$ as an ideal in the algebra $A$, so we need to show that it interacts well with the coproduct. That is, for each $x \in I$, we need to show that $\Delta(x) \in I \otimes A+A \otimes I$. It suffices to check this for the generators of the ideal; it is clear when $x=\xi_{m}$ with $m \leq j$, so let $x=\xi_{m}^{p^{n}}-\xi_{m}$, where $m>j$. We compute:

$$
\begin{aligned}
\Delta\left(\xi_{m}^{p^{n}}-\xi_{m}\right)= & \sum_{i=0}^{m}\left(\xi_{m-i}^{p^{n+i}} \otimes \xi_{i}^{p^{n}}-\xi_{m-i}^{p^{i}} \otimes \xi_{i}\right) \\
= & \left(\xi_{m}^{p^{n}}-\xi_{m}\right) \otimes 1+1 \otimes\left(\xi_{m}^{p^{n}}-\xi_{m}\right) \\
& +\sum_{i=1}^{m-1}\left(\left(\xi_{m-i}^{p^{n+i}}-\xi_{m-i}^{p^{i}}\right) \otimes \xi_{i}+\xi_{m-i}^{p^{n+i}} \otimes\left(\xi_{i}^{p^{n}}-\xi_{i}\right)\right) .
\end{aligned}
$$

Thus $\Delta\left(\xi_{m}^{p^{n}}-\xi_{m}\right)$ is in $I \otimes A+A \otimes I$, as desired.

As in [Rav86, Section 6.2], we can discuss the "topological linear dual" of the Hopf algebras $H_{j}^{n}$ : since $H^{n}$, for instance, is the direct limit of the finite-dimensional sub-Hopf algebras $B_{j}^{n}$, then the topological linear dual $\left(H^{n}\right)^{*}$ is defined to be $\left(H^{n}\right)^{*}=\lim _{j}\left(B_{j}^{n}\right)^{*}$, with the topology induced by the inverse limit.

The first part of the proof of Rav86, Theorem 6.2.3] applies in our setting to give the following.

Proposition 2.2. Fix $n \geq 1, j \geq 0$, and $\ell \in\{1,2, \ldots\} \cup\{\infty\}$, and let $H$ be the following sub-Hopf algebra of $H_{j}^{n} \otimes \mathbf{F}$ :

$$
H=\mathbf{F}\left[\xi_{j+1}, \ldots \xi_{j+\ell}\right] /\left(\xi_{i}^{p^{n}}-\xi_{i}: j+\ell \geq i \geq j+1\right) .
$$

Then the topological linear dual $H^{*}$ of $H$ is a group algebra.

For example, this applies when $H=B_{j}^{n} \otimes \mathbf{F}$ or $H=H_{j}^{n} \otimes \mathbf{F}$. By "the topological linear dual" of a finite-dimensional Hopf algebra like $B_{j}^{n}$, we mean its ordinary vector space dual with the discrete topology. 
We would like to identify the groups involved. Let $G^{n}, G_{j}^{n}$, and $P_{j}^{n}$ be the groups so that

$$
\begin{aligned}
& \mathbf{F}\left[G^{n}\right] \cong\left(H^{n} \otimes \mathbf{F}\right)^{*}, \\
& \mathbf{F}\left[G_{j}^{n}\right] \cong\left(H_{j}^{n} \otimes \mathbf{F}\right)^{*}, \\
& \mathbf{F}\left[P_{j}^{n}\right] \cong\left(B_{j}^{n} \otimes \mathbf{F}\right)^{*} .
\end{aligned}
$$

Of course, $G_{0}^{n}=G^{n}$ since $H_{0}^{n}=H^{n}$. Also, note that $P_{j}^{n}$ is a finite $p$-group and $G_{j}^{n}$ is a pro- $p$ group: $H_{j}^{n}$ is the direct limit of the finite-dimensional sub-Hopf algebras

$$
\mathbf{F}_{p}\left[\xi_{j+1}, \ldots, \xi_{j+\ell}\right] /\left(\xi_{i}^{p^{n}}-\xi_{i}\right),
$$

and this direct system becomes an inverse system of group algebras, induced by an inverse system of groups, after tensoring up with $\mathbf{F}$ and dualizing.

Proposition 2.3. Fix $n \geq 1$ and $j \geq 0$.

(a) $G^{n}$ is the group of strict automorphisms of the additive formal group law over $\mathbf{F}$. That is, $G^{n}$ is the group of power series of the form $x+\sum_{i \geq 1} a_{i} x^{p^{i}}$ with $a_{i} \in \mathbf{F}$, under composition.

(b) $G_{j}^{n}$ is the subgroup of $G^{n}$ consisting of such power series with $a_{i}=0$ for $1 \leq i \leq j$.

(c) $P_{j}^{n}$ is the quotient group $G^{n} / G_{j}^{n}$. That $i s, P_{j}^{n}$ is the group of power series of the form in (a), with $x^{p^{j+1}}=0$.

Proof. This follows from the well-known observation that $A$ corepresents the strict automorphisms of the additive formal group law for $\mathbf{F}_{p}$-algebras; see [Rav86, p. 378], for example. That is, $A$ is the $\bmod p$ group scheme for this strict automorphism group.

In more detail: we prove (c) first. $B_{j}^{n}$ is dual to the group algebra of a finite group, and the group is precisely the group of points in the group scheme defined by $B_{j}^{n} \otimes \overline{\mathbf{F}}_{p}$, where $\overline{\mathbf{F}}_{p}$ is the algebraic closure of $\mathbf{F}_{p}$ :

$$
P_{j}^{n}=\operatorname{Hom}_{\overline{\mathbf{F}}_{p} \text {-alg }}\left(B_{j}^{n} \otimes \overline{\mathbf{F}}_{p}, \overline{\mathbf{F}}_{p}\right) .
$$

By imitating the analysis for $A$, one can see that this is the group of power series of the form

$$
x+a_{1} x^{p}+a_{2} x^{p^{2}}+\cdots+a_{j} x^{p^{j}}
$$

under composition, subject to $x^{p^{j+1}}=0$, where $a_{i} \in \overline{\mathbf{F}}_{p}$ and $a_{i}^{p^{n}}=a_{i}$. In particular, $a_{i} \in \overline{\mathbf{F}}_{p}$ is the image of $\xi_{i}$. The elements $a$ of $\overline{\mathbf{F}}_{p}$ satisfying $a^{p^{n}}=a$ form a subfield isomorphic to $\mathbf{F}$, and this completes the proof of part (c).

To prove (a), take limits: since $H^{n}$ is the direct limit of the finite-dimensional sub-Hopf algebras $B_{j}^{n}, H^{n}$ represents the group scheme

$$
\operatorname{Hom}_{\overline{\mathbf{F}}_{p} \text {-alg }}\left(\underset{j}{\lim } B_{j}^{n} \otimes \overline{\mathbf{F}}_{p},-\right)=\lim _{j} \operatorname{Hom}_{\overline{\mathbf{F}}_{p} \text {-alg }}\left(B_{j}^{n} \otimes \overline{\mathbf{F}}_{p},-\right) \text {. }
$$

$G^{n}$ is the group of $\overline{\mathbf{F}}_{p}$-points in this group scheme, which is as described in (a).

The proof of part (b) is similar.

We end this section with the following result.

Proposition 2.4. Fix $n \geq 1$ and $j \geq 0$. Then $G_{j}^{n}$ is torsion-free. 
Proof. Fix $\alpha \in G_{j}^{n}$ different from the identity element, so $\alpha=x+\sum_{i>j} a_{i} x^{p^{i}}$ with at least one $a_{i}$ nonzero. Indeed, suppose that $a_{m}$ is the first nonzero coefficient. Then we claim that in $\alpha^{p}$, the coefficient of $x^{p^{p m}}$ is the first nonzero term. This implies that the elements $\left\{\alpha^{p^{i}} \mid i \geq 0\right\}$ are distinct, and so $\alpha$ is not a torsion element.

To verify the claim, we need to know the coefficient of $x^{p^{i}}$ in $\alpha^{p}$. Since the product in $G_{j}^{n}$ is determined by the coproduct in $H_{j}^{n}$, to find any coefficient in $\alpha^{p}$, we use the $p$ th iterated coproduct in $H_{j}^{n}$, which is defined in Notation 2.5 and a formula for which is given in Lemma 2.6. The $p$ th iterated coproduct on $\xi_{p m}$ has a term

$$
\underbrace{\xi_{m}^{p^{(p-1) m}} \otimes \cdots \otimes \xi_{m}^{p^{2 m}} \otimes \xi_{m}^{p^{m}} \otimes \xi_{m}}_{p \text { factors }}
$$

which produces the nonzero term

$$
a_{m}^{p^{(p-1) m}+p^{(p-2) m}+\cdots+p^{2 m}+p^{m}+1}
$$

in the coefficient of $x^{p m}$. Since $a_{m}$ is the first nonzero coefficient in $\alpha$, then only terms in the iterated coproduct having no $\xi_{i}$ factors with $0<i<m$ are relevant. So all of the other relevant terms in the $p$ th iterated coproduct of $\xi_{p m}$, and all of the relevant terms in the $p$ th iterated coproduct of $\xi_{k}$ for $k<p m$, will have at least one tensor factor equal to 1 , and hence will occur with some multiplicity. It is easy to check that the multiplicity is divisible by $p$ (see Lemma 2.7) and hence is zero in $\mathbf{F}$.

Notation 2.5. Given a coassociative coalgebra $C$ with coproduct $\Delta$, we let $\Delta^{2}=$ $\Delta$, and $\Delta^{m}=\left(\Delta \otimes 1^{\otimes m-2}\right) \circ \Delta^{m-1}$ for $m \geq 3$; we call $\Delta^{m}$ the " $m$ th iterated coproduct". This is indexed so that $\Delta^{m}(x)$ lies in $C^{\otimes m}$.

The following is immediate from the formula for the coproduct in $H_{j}^{n}$.

Lemma 2.6. For any $m \geq 1$, the mth iterated coproduct in $H_{j}^{n}$ of $\xi_{\ell}$ is

$$
\Delta^{m}\left(\xi_{\ell}\right)=\sum_{\substack{i_{1}+\cdots+i_{m}=\ell \\ i_{k}=0 \text { or } i_{k}>j}} \xi_{i_{m}}^{p^{i_{1}+\cdots+i_{m-1}}} \otimes \cdots \otimes \xi_{i_{3}}^{p^{i_{1}+i_{2}}} \otimes \xi_{i_{2}}^{p^{i_{1}}} \otimes \xi_{i_{1}} .
$$

Lemma 2.7. Given an ordered list $\left(b_{1}, \ldots, b_{m}\right)$ and a number $p \geq m$, then the number of ways of adding $p-m 1$ 's to the list, while preserving the ordering of the $b_{i}$ 's, is $\left(\begin{array}{c}p \\ m\end{array}\right)$.

The proof is a simple combinatorial argument.

Thus given a $p$ th iterated coproduct with $p-m$ 1's in it, it occurs with multiplicity $\left(\begin{array}{c}p \\ m\end{array}\right)$. If $p$ is prime and $0<m<p$, this multiplicity is congruent to zero $\bmod p$.

\section{EXAMPLES AND OBSERVATIONS}

In this section, we collect some miscellaneous observations about the Hopf algebras $H_{j}^{n}$ and the corresponding groups $G_{j}^{n}$.

Example 3.1 $\left(H^{1}\right)$. Let $n=1$. In this case, the Hopf algebra $H^{1}$ is bicommutative. Avinoam Mann pointed out (private communication) that the corresponding abelian group $G^{1}$ is isomorphic to the group of power series of the form $1+\sum a_{i} x^{i}$ with multiplication as the group operation. According to Cam00, p. 218], this group is a free abelian pro- $p$ group of infinite rank. In particular, it is not $p$-adic 
analytic, since such groups must have finite rank. Since $G^{1}$ is a subgroup of $G^{n}$ for every $n$, no $G^{n}$ is $p$-adic analytic.

One might also note that for any $n$, the group $G^{n}$ is a subgroup of the Nottingham group $\mathcal{N}=\mathcal{N}(\mathbf{F})$, which consists of all power series $x+\sum_{j \geq 2} b_{j} x^{j}$ with $b_{j} \in \mathbf{F}$, under composition. Such an observation is almost a triviality, since every finite $p$-group, and every countably-based pro $p$-group, can be embedded as a subgroup of $\mathcal{N}$; the group $G^{n}$ is embedded in a particularly nice way, though - it is an "index subgroup". See [Cam00, Theorems 10 and 11, and pp. 217-218] for more on the Nottingham group.

Example 3.2 $\left(P_{j}^{1}\right.$ and $\left.G^{1}\right)$. The group $P_{j}^{n}$ corresponding to the Hopf algebra $B_{j}^{n}$ is a finite $p$-group of order $p^{n j}$. $P_{1}^{n}$ is abelian for all $n$; indeed, $P_{1}^{n}$ is isomorphic to the additive group of $\mathbf{F}=\mathbf{F}_{p^{n}}: P_{1}^{n} \cong\left(C_{p}\right)^{n}$. (We write $C_{d}$ for the cyclic group of order $d$.) For each $j, P_{j}^{1}$ is abelian of order $p^{j}$, and one can show that

$$
P_{j}^{1} \cong \prod_{i \leq j, p \nmid i} C_{\alpha_{p}\left(\frac{j+1}{i}\right)}
$$

where $\alpha_{p}(r)$ is the smallest power of $p$ greater than or equal to $r$ :

$$
\alpha_{p}(r)=\min _{i}\left\{p^{i}: p^{i} \geq r\right\}=p^{\left\lceil\log _{p}(r)\right\rceil} .
$$

In this decomposition, the cyclic summand of order $\alpha_{p}\left(\frac{j+1}{i}\right)$ is generated by the power series $1+x^{i}$. Thus in the inverse system

$$
\cdots \rightarrow P_{j+2}^{1} \rightarrow P_{j+1}^{1} \rightarrow P_{j}^{1} \rightarrow \cdots
$$

defining $G^{1}$, the cyclic summands at the $j$ th stage are the images from the later stages of cyclic summands of larger and larger orders. Of course this must happen, since $G^{1}$ is torsion-free; in this particular case, though, we can observe it directly.

Example 3.3 (A filtration). Note that $B_{j}^{n}$ is a sub-Hopf algebra of $B_{j+1}^{n}$, and the (conormal) quotient is $\mathbf{F}_{p}\left[\xi_{j}\right] /\left(\xi_{j}^{p^{n}}-\xi_{j}\right)$ with $\xi_{j}$ primitive. This quotient is isomorphic to $B_{1}^{n}$. Dually, for each $j \geq 1$ there is a group extension

$$
1 \rightarrow\left(C_{p}\right)^{n} \rightarrow P_{j+1}^{n} \rightarrow P_{j}^{n} \rightarrow 1 .
$$

$G^{n}$ is the inverse limit of the groups $P_{j}^{n}$ with respect to these surjections. So the associated graded consists of a copy of $P_{1}^{n}=\left(C_{p}\right)^{n}=\mathbf{F}$ in each degree.

Equivalently, since $G_{j}^{n}$ is the kernel of the map $G^{n} \rightarrow P_{j}^{n}, G_{j}^{n}$ is normal in $G^{n}$. Indeed, each $G_{j}^{n}$ is normal in $G_{k}^{n}$ whenever $j \geq k$. This gives a filtration of $G^{n}$ :

$$
G^{n}=G_{0}^{n} \supseteq G_{1}^{n} \supseteq G_{2}^{n} \supseteq \cdots .
$$

Again, the associated graded is isomorphic to $\mathbf{F}$ in each degree.

We also point out that whenever $n$ divides $m, \mathbf{F}_{p^{n}}$ is a subfield of $\mathbf{F}_{p^{m}}$, so $G^{n}$ is a subgroup of $G^{m}$. For the same reason, the colimit of the groups $G^{n}$ is the group of strict automorphisms of the additive formal group over $\overline{\mathbf{F}}_{p}$, which we denote by $G^{\infty}$. One can define $G_{j}^{\infty}$ analogously to $G_{j}^{n}: G_{j}^{\infty}$ consists of power series in $G^{\infty}$ with the first $j$ coefficients equal to zero. Summarizing, we have the following.

Proposition 3.4. $\quad$ (a) Fix $j \geq 0$ and $k, n \geq 1$. Then $G_{j}^{n}$ is a subgroup of $G_{j}^{k n}$. Dually, $H_{j}^{n}$ is a quotient of $H_{j}^{k n}$ : the map sending $\xi_{i} \in H_{j}^{k n}$ to $\xi_{i} \in H_{j}^{n}$ is a surjective Hopf algebra map. 
(b) Fix $j \geq 0$. Then with respect to the inclusions in part (a),

$$
\underset{n}{\lim _{n}} G_{j}^{n} \cong G_{j}^{\infty} .
$$

As far as dualizing part (b), see Proposition 5.4 for a partial result.

Note that it is not necessary to tensor up to $\mathbf{F}_{p^{k n}}$ coefficients to get the quotient map $H_{j}^{k n} \rightarrow H_{j}^{n}$. We also point out that these subgroups are not normal:

Proposition 3.5. Fix $n \geq 1$. $G^{n}$ is not normal in $G^{k n}$ if $k \geq 2$. Thus $H^{n}$ is not a conormal quotient of $H^{k n}$ if $k \geq 2$; that is, $H^{k n} \square_{H^{n}} \mathbf{F}_{p}$ and $\mathbf{F}_{p} \square_{H^{n}} H^{k n}$ are different as sub-vector spaces of $H^{k n}$.

In order to prove this, we need to know how to invert elements of $G^{k n}$. We recall the formula for the antipode in $A$ from Mil58. Let Part $(n)$ denote the set of ordered partitions of $n$ (so, for example, $(3,1)$ and $(1,3)$ are distinct elements of $\operatorname{Part}(4))$. Given an ordered partition $\alpha \in \operatorname{Part}(n)$, write $\ell(\alpha)$ for the length of $\alpha$; for $i \leq \ell(\alpha)$, let $\alpha(i)$ be the $i$ th term of $\alpha$, and let $\sigma(i)$ be the sum of the first $i-1$ terms of $\alpha$.

Lemma 3.6 (Lemma 10 in Mil58]). The conjugate of $\xi_{n}$ in $A$ is equal to

$$
\chi\left(\xi_{n}\right)=\sum_{\alpha \in \operatorname{Part}(n)}(-1)^{\ell(\alpha)} \prod_{i=1}^{\ell(\alpha)} \xi_{\alpha(i)}^{p^{\sigma(i)}} .
$$

The same formula applies in $H_{j}^{n}$ and $B_{j}^{n}$ for all $n$ and $j$.

In particular, the summand corresponding to the partition $(1,1, \ldots, 1)$ is

$$
(-1)^{n} \xi_{1}^{1+p+p^{2}+\cdots+p^{n-1}} \text {. }
$$

Proof of Proposition 3.5. Fix $n \geq 1$ and $k \geq 2$. To see that $G^{n}$ is not normal in $G^{k n}$, conjugate $\alpha=x+x^{p} \in G^{1} \leq G^{n}$ by $\beta=x+b_{1} x^{p}$ for some $b_{1} \in \mathbf{F}_{p^{k n}} \backslash \mathbf{F}_{p^{n}}$. Using Lemma 3.6 and the observation following it, one can see that

$$
\beta^{-1}=x+\sum_{i=1}^{\infty}(-1)^{i} b_{1}^{1+p+\cdots+p^{i-1}} x^{p^{i}} .
$$

Therefore

$$
\begin{aligned}
\alpha \beta^{-1} & =\beta^{-1}+\left(\beta^{-1}\right)^{p} \\
& =x+\left(-b_{1}+1\right) x^{p}+\sum_{i=2}^{\infty}(-1)^{i}\left(b_{1}^{1+p+\cdots+p^{i-1}}-b_{1}^{p+p^{2}+\cdots+p^{i-1}}\right) x^{p^{i}} .
\end{aligned}
$$

So one can compute $\beta \alpha \beta^{-1}$ :

$$
\begin{aligned}
\beta \alpha \beta^{-1} & =\left(\alpha \beta^{-1}\right)+b_{1}\left(\alpha \beta^{-1}\right)^{p} \\
& =x+x^{p}+\left(-b_{1}^{p}+b_{1}\right)\left(x^{p^{2}}-b_{1} x^{p^{3}}+b_{1}^{1+p} x^{p^{4}}-b_{1}^{2+p} x^{p^{5}}+\cdots\right) .
\end{aligned}
$$

We are assuming that $b_{1} \notin \mathbf{F}_{p^{n}}$, so $b_{1}^{p} \neq b_{1}$. If the coefficient $-b_{1}^{p}+b_{1}$ of $x^{p^{2}}$ is in $\mathbf{F}_{p^{n}}$, then the coefficient $\left(-b_{1}^{p}+b_{1}\right) b_{1}$ of $x^{p^{3}}$ is not. So $\beta \alpha \beta^{-1}$ is not in $G^{n}$, although $\alpha$ is. 


\section{The COHOMOlOGical VARIETY of $H^{n}$ IS TRIVIAL}

Let $H^{n}$ be as above. In this section, we examine the cohomology of $H^{n}$. By "cohomology," we mean $\operatorname{Ext}_{H^{n}}^{*}\left(\mathbf{F}_{p}, \mathbf{F}_{p}\right)$, where Ext is computed in the category of $H^{n}$-comodules. The main result is that every positive dimensional cohomology class is nilpotent.

Since after tensoring up with $\mathbf{F}_{p^{n}}$, the topological linear dual of $H^{n}$ is the group algebra of the profinite group $G^{n}$, we have the following result, which is analogous to [Rav86, Corollary 6.2.4]. First note that any $H^{n}$-comodule $M$ may be viewed as an $\left(H^{n}\right)^{*}$-module via the composite

$$
\left(H^{n}\right)^{*} \otimes M \rightarrow\left(H^{n}\right)^{*} \otimes H^{n} \otimes M \rightarrow M .
$$

This makes $M \otimes \mathbf{F}_{p^{n}}$ into a discrete $G^{n}$-module.

Proposition 4.1. There is an isomorphism

$$
\operatorname{Ext}_{H^{n}}^{*}\left(\mathbf{F}_{p}, \mathbf{F}_{p}\right) \otimes \mathbf{F}_{p^{n}} \cong H_{c}^{*}\left(G^{n}, \mathbf{F}_{p^{n}}\right),
$$

where $H_{c}^{*}$ denotes continuous group cohomology.

(There should be a similar result for cohomology with coefficients in any $H^{n}$ comodule $M$, but we are only interested in the case when $M$ is the trivial comodule.)

Proof. Since $G^{n}$ is the inverse limit of the finite groups $P_{j}^{n}, H_{c}^{*}\left(G^{n}, \mathbf{F}_{p^{n}}\right)$ may be defined as follows (see [Ser02, I.2.2, Proposition 8]):

$$
H_{c}^{*}\left(G^{n}, \mathbf{F}_{p^{n}}\right)=\underset{j}{\lim } H^{*}\left(P_{j}^{n}, \mathbf{F}_{p^{n}}\right) .
$$

Since $P_{j}^{n}$ is finite and its group algebra is dual to $B_{j}^{n}$, we have an isomorphism $H^{*}\left(P_{j}^{n},-\right) \cong \operatorname{Ext}_{B_{j}^{n} \otimes \mathbf{F}_{p^{n}}}^{*}\left(\mathbf{F}_{p^{n}},-\right)$. The result follows from the observation that $H^{n}=\lim _{\text {促 }} B_{j}^{n}$

We also need the following. Here, $\otimes$ means $\otimes_{k}$.

Lemma 4.2. Let $C$ be a coaugmented coalgebra over a field $k$, and let $F$ be a field extension of $k$. Then there is an isomorphism of algebras $\operatorname{Ext}_{C \otimes F}^{*}(F, F) \cong$ $\operatorname{Ext}_{C}^{*}(k, k) \otimes F$.

Proof. Take an injective resolution of $k$ as a $C$-comodule, and tensor with $F$. The result is an injective resolution of $k \otimes F=F$ as a $C \otimes F$-comodule.

Here is the main result of this section.

Theorem 4.3. Every positive-dimensional element in $\operatorname{Ext}_{H^{n}}^{*}\left(\mathbf{F}_{p}, \mathbf{F}_{p}\right)$ is nilpotent.

Proof. As above, let $\mathbf{F}=\mathbf{F}_{p^{n}} . H^{n} \otimes \mathbf{F}$ is dual to the group algebra $\mathbf{F}\left[G^{n}\right]$, where $G^{n}$ is the group defined before Proposition 2.3. By Proposition 4.1. $\left.\operatorname{Ext}_{H^{n} \otimes \mathbf{F}}^{*} \mathbf{F}, \mathbf{F}\right) \cong$ $H_{c}^{*}\left(G^{n} ; \mathbf{F}\right)$. Proposition 2.4 says that $G^{n}$ is torsion-free, and so Qui71, Proposition 13.4] applies, to say that every positive-dimensional element in $\operatorname{Ext}_{H^{n} \otimes \mathbf{F}}^{*}(\mathbf{F}, \mathbf{F})$ is nilpotent. Hence by Lemma 4.2, the same is true for $\operatorname{Ext}_{H^{n}}^{*}\left(\mathbf{F}_{p}, \mathbf{F}_{p}\right)$.

Given the description of the group $G^{1}$ in Example 3.1, we expect these Ext algebras to be infinitely generated. This contrasts with the situation for Morava stablizer groups, some of which are Poincaré duality groups, and all of which have 
reasonably well-behaved cohomology: Rav86, Theorem 6.2.10] says that the cohomology of $S^{n}$ is always finitely generated, and either satisfies Poincare duality or is periodic.

Question 4.4. When $n=1$, the corresponding group is a free abelian pro- $p$ group, and so every element in its cohomology has nilpotence height $p$. Is there a uniform nilpotence height for the cohomology of $H^{n}$ when $n \geq 2$ ?

\section{On the cohomology of the complete Steenrod algebra}

As we discussed in the Introduction, the results in this paper arose in part as an attempt to study the cohomology of the complete Steenrod algebra. In this section, we explore this algebra, its cohomology, and the relationship to $H^{n}$.

We start by recalling from May70 the relationship between the Frobenius map on a commutative Hopf algebra and the Steenrod operation $\widetilde{\mathscr{P}}^{0}$ on Ext. As usual, Ext is computed in the category of comodules.

Let $B$ be a graded commutative Hopf algebra over $\mathbf{F}_{p}$. By May's work May70, one has Steenrod operations acting on $\operatorname{Ext}_{B}^{*}\left(\mathbf{F}_{p}, \mathbf{F}_{p}\right)$. In this paper, we are only interested in the operation called $\widetilde{\mathscr{P}}^{0}$ (also called $\widetilde{S q}$ when $p=2$ ), which is an operation

$$
\widetilde{\mathscr{P}}^{0}: \operatorname{Ext}_{B}^{s, t}\left(\mathbf{F}_{p}, \mathbf{F}_{p}\right) \rightarrow \operatorname{Ext}_{B}^{s, p t}\left(\mathbf{F}_{p}, \mathbf{F}_{p}\right) .
$$

Proposition 5.1 (Proposition 11.10 in [May70). Let B be a graded connected commutative Hopf algebra over the field $\mathbf{F}_{p}$. The Frobenius map $\phi: b \mapsto b^{p}$ is a Hopf algebra map, and the induced map $\phi_{*}$ on Ext is the Steenrod operation $\widetilde{P^{0}}$.

We defined the dual complete Steenrod algebra $\widehat{A}$ in the Introduction:

$$
\widehat{A}=\lim _{\longrightarrow}\left(\mathbf{F}_{p}\left[\xi_{n}: n \geq 1\right] \rightarrow \mathbf{F}_{p}\left[\xi_{n}^{p^{-1}}: n \geq 1\right] \rightarrow \mathbf{F}_{p}\left[\xi_{n}^{p^{-2}}: n \geq 1\right] \rightarrow \cdots\right),
$$

with the coproduct inherited from A. Arnon Arn94, Arn00] defined the complete Steenrod algebra, and we note that it is dual to $\widehat{A}$. (This is also the assertion in [LH96, Definition 3.6].)

Proposition 5.2. The dual $\widehat{A}^{*}$ of $\widehat{A}$ is the complete Steenrod algebra.

Proof. The dual complete Steenrod algebra $\widehat{A}$ is defined as a direct limit. The complete Steenrod algebra $\widehat{A}^{*}$ is defined in [Arn00, p. 185] as the dual inverse limit.

$\widehat{A}$ inherits a grading from $A$ : $\xi_{n}^{p^{k}}$ is in degree $2 p^{k}\left(p^{n}-1\right)$ when $p$ is odd, $2^{k}\left(2^{n}-1\right)$ when $p=2$. Thus $\widehat{A}$ is a $\mathbf{Z}\left[\frac{1}{p}\right]$-graded Hopf algebra. It is not finite-dimensional in each grading, though, so while the monomial basis is a pleasant one for $\widehat{A}$, there is no nice basis for its dual. Furthermore, the dual is not graded anymore, strictly speaking. In the language of [LH96, Definition 1.1], $\widehat{A}^{*}$ is a "large" $\mathbf{Z}\left[\frac{1}{p}\right]$-graded algebra - additively, it is not isomorphic to the direct sum of its homogeneous pieces, but rather is a (proper) subgroup of the product of those pieces.

As far as we can tell, the cohomology of the complete Steenrod algebra has not been studied in any published paper. 
Proposition 5.3. The cohomology of $\widehat{A}$ is isomorphic to the cohomology of $A$ with the operation $\widetilde{\mathscr{P}}^{0}$ inverted:

$$
\operatorname{Ext}_{\widehat{A}}^{*}\left(\mathbf{F}_{p}, \mathbf{F}_{p}\right) \cong\left(\widetilde{\mathscr{P}}^{0}\right)^{-1} \operatorname{Ext}_{A}^{*}\left(\mathbf{F}_{p}, \mathbf{F}_{p}\right) .
$$

Proof. We may rewrite the direct limit defining $\widehat{A}$ as follows:

$$
\widehat{A}=\underline{\lim }(A \stackrel{\phi}{\rightarrow} A \stackrel{\phi}{\rightarrow} \cdots) .
$$

Coalgebra cohomology commutes with direct limits, so take Ext of this directed system:

$$
\operatorname{Ext}_{\widehat{A}}^{*}\left(\mathbf{F}_{p}, \mathbf{F}_{p}\right)=\underline{\lim }\left(\operatorname{Ext}_{A}^{*}\left(\mathbf{F}_{p}, \mathbf{F}_{p}\right) \stackrel{\phi_{*}}{\longrightarrow} \operatorname{Ext}_{A}^{*}\left(\mathbf{F}_{p}, \mathbf{F}_{p}\right) \stackrel{\phi_{*}}{\longrightarrow} \cdots\right) .
$$

Proposition 5.1 says that $\phi_{*}=\widetilde{\mathscr{P}}^{0}$, and this finishes the proof.

As mentioned in the Introduction, much of the work in this paper was motivated, directly or indirectly, by Conjecture 1.4. which is equivalent to the statement that every positive degree element in $\operatorname{Ext}_{\widehat{A}}^{*}\left(\mathbf{F}_{p}, \mathbf{F}_{p}\right)$ is nilpotent. Theorem 1.2 says that this is true when $p=2$.

For example, let $p=2$. There is a family of cohomology classes $\left\{h_{1, k}: k \in \mathbf{Z}\right\}$ in the cohomology of $\widehat{A}$, represented in the cobar complex by $\left[\xi_{1}^{k}\right]$. The classes with $k \geq 0$ lift to classes of the same name in $\operatorname{Ext}_{A}^{*}\left(\mathbf{F}_{2}, \mathbf{F}_{2}\right)$, and in the cohomology of $A, h_{1,0}$ is nonnilpotent-its powers produce the spike in the zero stem at the $E_{2}$-term of the Adams spectral sequence - while $h_{1, k}$ is nilpotent when $k \geq 1$. In particular, $h_{1, k}^{4}=0$ for all $k \geq 1$. These classes are connected by the algebra map $\widetilde{S q}^{0}: \widetilde{S q}^{0}\left(h_{1, k}\right)=h_{1, k+1}$, so if $h_{1, k}$ is nilpotent, so is $h_{1, k+1}$. In $\operatorname{Ext}_{\widehat{A}^{*}}^{*}\left(\mathbf{F}_{2}, \mathbf{F}_{2}\right), \widetilde{S q}^{0}$ has been inverted, so the relation $h_{1,1}^{4}=0$ implies that $h_{1, k}^{4}=0$ for all $k \in \mathbf{Z}$.

The multiplicative structure in the cohomology of the polynomial part of the odd primary Steenrod algebra is less well-understood. For example, there are classes $h_{1, k} \in \operatorname{Ext}_{A}^{1}\left(\mathbf{F}_{p}, \mathbf{F}_{p}\right)$, for $k \geq 0$, represented in the cobar complex by $\left[\xi_{1}^{p^{k}}\right]$. These classes are odd dimensional, so by graded commutativity of Ext, they square to zero. There are related classes $b_{1, k} \in \mathrm{Ext}^{2}$, though, which can be obtained by applying the Bockstein operation to $h_{1, k}$; equivalently, $b_{1, k}$ is represented in the cobar complex by

$$
\sum_{i=1}^{p-1} \frac{1}{p}\left(\begin{array}{c}
p \\
i
\end{array}\right)\left[\xi_{1}^{i p^{k}} \mid \xi_{1}^{(n-i) p^{k}}\right] .
$$

One can show that $b_{1,0}$ is nonnilpotent, in analogy with $h_{1,0}$ at the prime 2 . The nilpotence (or lack thereof) of $b_{1, k}$ for $k>0$ has not been determined in general; a result of Nakamura [Nak75, Proposition 1.1(c)] shows that these classes are all nilpotent when $p=3$, and this is the best known result in this direction.

Given Conjecture 1.4. one could conclude that $b_{1, k}$ is nilpotent in $\operatorname{Ext}_{\widehat{A}}^{*}\left(\mathbf{F}_{p}, \mathbf{F}_{p}\right)$, and hence $b_{1, k}$ is nilpotent in $\operatorname{Ext}_{A}^{*}\left(\mathbf{F}_{p}, \mathbf{F}_{p}\right)$ for sufficiently large $k$. Then Pal01, Lemma B.3.3] would imply that $b_{1, k}$ is nilpotent for all $k \geq 1$. In general, a positive solution to Conjecture 1.4 would be a helpful step in trying to prove odd primary analogues of the nilpotence and $F$-isomorphism results in Pal99. See [Pal01, Conjecture 5.4.1] and the surrounding discussion.

We end this section by exploring the connection between the dual complete Steenrod algebra $\widehat{A}$ and the Hopf algebras $H^{n}$, with an eye toward Conjecture 1.4. 
The philosophy is that, while $\widehat{A}$ is defined by formally inverting the Frobenius $\phi, H^{n}$ is defined by forcing $\phi^{n}$ to be the identity. At the level of cohomology, $\widetilde{\mathscr{P}^{0}}$ is invertible of infinite order in $\operatorname{Ext}_{\widehat{A}}^{*}\left(\mathbf{F}_{p}, \mathbf{F}_{p}\right)$, and it is invertible of order $n$ in $\operatorname{Ext}_{H^{n}}^{*}\left(\mathbf{F}_{p}, \mathbf{F}_{p}\right)$. So one can hope that knowing that every positive-dimensional element in $\operatorname{Ext}_{H^{n}}^{*}\left(\mathbf{F}_{p}, \mathbf{F}_{p}\right)$ is nilpotent would be relevant to studying nilpotence of classes in $\operatorname{Ext}_{\widehat{A}}^{*}\left(\mathbf{F}_{p}, \mathbf{F}_{p}\right)$.

More formally, we start by noting that one can generalize the definition of $\widehat{A}$; in keeping with the definition and notation for $H_{j}^{n}$ from Section 2 for any $j \geq 0$ we let

$\widehat{A}_{j}=\lim _{\longrightarrow}\left(\mathbf{F}_{p}\left[\xi_{n}: n \geq j+1\right] \rightarrow \mathbf{F}_{p}\left[\xi_{n}^{p^{-1}}: n \geq j+1\right] \rightarrow \mathbf{F}_{p}\left[\xi_{n}^{p^{-2}}: n \geq j+1\right] \rightarrow \cdots\right)$.

Thus $\widehat{A}_{0}=\widehat{A}$, and $\widehat{A}_{j}$ is a conormal quotient Hopf algebra of $\widehat{A}_{k}$ whenever $j \geq k$. Note that for each $n$ and $j$, one can view $H_{j}^{n}$ as being a quotient of $\widehat{A}_{j}$ :

$$
H_{j}^{n}=\widehat{A}_{j} /\left(\xi_{i}^{p^{n+k}}-\xi_{i}^{p^{k}}: i \geq 1, k \in \mathbf{Z}\right) .
$$

Also, as noted in Proposition 3.4, $H_{j}^{n}$ is a quotient of $H_{j}^{m}$ whenever $n$ divides $m$. Thus for fixed $j$, the Hopf algebras $H_{j}^{n}$ form an inverse system.

Proposition 5.4. Fix $j \geq 0$. $\widehat{A}_{j}$ embeds in the inverse limit of the Hopf algebras $H_{j}^{n}$. This embedding is not an isomorphism.

Proof. The quotient maps $\widehat{A}_{j} \rightarrow H_{j}^{n}$ induce a map from $\widehat{A}_{j}$ to the inverse limit, and one can see that the kernel of the map to the limit is zero.

Let $H_{j}^{\infty}$ be the inverse limit. To see that $\widehat{A}_{j}$ is not isomorphic to $H_{j}^{\infty}$, we note that there are elements in $H_{j}^{\infty}$ of unipotence height $p$ : elements $x$ different from 1 satisfying $x^{p}=x$. To construct these elements, it suffices to construct a family of such elements, one in each $H_{j}^{n}$, mapping to each other in the inverse system. The relevant elements are $\xi_{i}^{p^{n}-1}$, for each $i>j$-computations show that $\left(\xi_{i}^{p^{n}-1}\right)^{p}=\xi_{i}^{p^{n}-1}$, and that $\xi_{i}^{p^{n d}-1}$ in $H^{n d}$ maps to $\xi_{i}^{p^{n}-1}$ in $H^{n}$.

Question 5.5. (a) What does this imply about the cohomology of $\widehat{A}$ ?

(b) What is the cokernel of the map $\widehat{A}_{j} \hookrightarrow \lim _{H_{j}^{n}}$ ?

(c) How are $H_{j}^{\infty}$ and $G_{j}^{\infty}$ (from Proposition 3.4) related? How are $\widehat{A}_{j}$ and $G_{j}^{\infty}$ related?

\section{Appendix A. Proof of Theorem 1.2}

We restate the theorem.

Theorem 1.2, Let $p=2$ and fix $z \in \operatorname{Ext}_{A}^{s, t}\left(\mathbf{F}_{2}, \mathbf{F}_{2}\right)$. If $s$ is positive, then there is an $n$ so that $\left(\widetilde{S q}^{0}\right)^{n}(z)$ is nilpotent.

Proof. This follows from the main result of Pal99.

In detail, for each pair of integers $(S, T)$ with $0 \leq S<T$, let $R_{T, S}$ be the polynomial algebra

$$
R_{T, S}=\mathbf{F}_{2}\left[h_{t s}: 0 \leq s \leq S<T \leq t\right]
$$


Let $R$ be the inverse limit of the $R_{T, S}$ under the apparent maps (map each polynomial generator to the generator of the same name if present, and to zero otherwise). Then

$$
R=\mathbf{F}_{2}\left[h_{t s}: s<t\right] /\left(h_{t s} h_{v u}: u \geq t\right) .
$$

Also, $R_{n, n-1}$ is the cohomology of this quotient Hopf algebra of $A$ :

$$
E(n)=A /\left(\xi_{1}, \ldots, \xi_{n-1}, \xi_{i}^{2^{n}}: i \geq n\right),
$$

with the polynomial generator $h_{t s}$ corresponding to the cobar element $\left[\xi_{t}^{2^{s}}\right]$. So there is a restriction map

$$
\rho^{n}: \operatorname{Ext}_{A}^{*}\left(\mathbf{F}_{2}, \mathbf{F}_{2}\right) \rightarrow R_{n, n-1} .
$$

These can be assembled to give a map

$$
\rho: \operatorname{Ext}_{A}^{*}\left(\mathbf{F}_{2}, \mathbf{F}_{2}\right) \rightarrow R
$$

and Theorems 1.3 and 1.4 of Pal99] say that up to nilpotence, $\rho$ is an isomorphism between the cohomology of $A$ and a certain subring of the codomain. In particular, $\rho$ is a monomorphism mod nilpotence.

Since $\rho$ is the inverse limit of the maps $\rho^{n}$, and since each $\rho^{n}$ is a restriction map, $\rho$ commutes with the action of $\widetilde{S q}^{0}$. Hence there is an induced map

$$
\rho:\left(\widetilde{S q}^{0}\right)^{-1} \operatorname{Ext}_{A}^{*}\left(\mathbf{F}_{2}, \mathbf{F}_{2}\right) \rightarrow\left(\widetilde{S q}^{0}\right)^{-1} R,
$$

which is a monomorphism mod nilpotence. But the codomain is zero- the Frobenius map acts nilpotently on each element of the Hopf algebra $E(n)$. Equivalently, at the level of cohomology, there is a multiplicative action of $\widetilde{S q}^{0}$ on $R$, defined by $\widetilde{S q}^{0}\left(h_{t s}\right)=h_{t, s+1}$ if $s+1<t$, but $\widetilde{S q}^{0}\left(h_{t, t-1}\right)=0$. Thus for each element $y \in R$, $\left(\widetilde{S q}^{0}\right)^{n}(y)=0$ for $n$ sufficiently large.

\section{ACKNOWLEDGMENTS}

Much of the work on the Hopf algebras $H^{n}$ was inspired by a brief conversation with Mike Hopkins. I have had many helpful discussions with Ethan Devinatz and Hal Sadofsky. Also, several of the results and proofs owe an obvious debt to Section 6.2 of Ravenel's green book Rav86].

\section{REFERENCES}

[Arn94] D. Arnon, Generalized Dickson invariants, Ph.D. thesis, Mass. Inst. of Tech., 1994.

[Arn00] , Generalized Dickson invariants, Israel J. Math. 118 (2000), $183-205$. MR,2001i:55020

[Cam00] R. Camina, The Nottingham group, New horizons in pro-p groups, Birkhäuser Boston, Boston, MA, 2000, pp. 205-221. MR2001f:20054

[LH96] I. Llerena and N. H. V. Hu'ng, The complete Steenrod algebra and the generalized Dickson algebra, Algebraic topology: new trends in localization and periodicity (Sant Feliu de Guíxols, 1994), Birkhäuser, Basel, 1996, pp. 271-284. MR.97i:55032

[May70] J. P. May, A general algebraic approach to Steenrod operations, The Steenrod Algebra and its Applications (Proc. Conf. to Celebrate N. E. Steenrod's Sixtieth Birthday, Battelle Memorial Inst., Columbus, Ohio, 1970) (F. P. Peterson, ed.), Lecture Notes in Mathematics, Vol. 168, Springer, Berlin, 1970, pp. 153-231. MR43:6915

[Mil58] J. W. Milnor, The Steenrod algebra and its dual, Ann. of Math. (2) 67 (1958), $150-171$. MR.20:6092

[Nak75] O. Nakamura, Some differentials in the mod 3 Adams spectral sequence, Bull. Sci. Engrg. Div. Univ. Ryukyus Math. Natur. Sci. (1975), no. 19, 1-25. MR.52:6711 
[Pal99] J. H. Palmieri, Quillen stratification for the Steenrod algebra, Ann. of Math. (2) 149 (1999), no. 2, 421-449. MR2000g:55026

[Pal01] J. H. Palmieri, Stable homotopy over the Steenrod algebra, Mem. Amer. Math. Soc. 151 (2001), no. 716, xiv+172. MR2002a:55019

[Qui71] D. G. Quillen, The spectrum of an equivariant cohomology ring. I, II, Ann. of Math. (2) 94 (1971), 549-572, 573-602. MR45:7743

[Rav86] D. C. Ravenel, Complex cobordism and stable homotopy groups of spheres, Pure and Applied Mathematics, vol. 121, Academic Press Inc., Orlando, FL, 1986. MR87j:55003

[Ser02] J.-P. Serre, Galois cohomology, English ed., Springer Monographs in Mathematics, Springer-Verlag, Berlin, 2002, Translated from the French by Patrick Ion and revised by the author. MR2002i:12004

Department of Mathematics, University of Washington, Box 354350, Seattle, WashINGTON 98195-4350

E-mail address: palmieri@math.washington.edu 